\title{
Oscillation Criteria for Certain Second Order Difference Equations
}

\author{
E. THANDAPANI
}

Some oscillation criteria for the second order nonlinear difference equations

$$
\Delta^{2} y_{n-1}+\sum_{j=1}^{m} q_{i n} f_{i}\left(y_{n}\right)_{i j}\left(\Delta y_{n-1}\right)=0 \quad(n \in \mathbb{N})
$$

are establishod.

Key words: Oscillation, second order differenco equations

AMS subject classification: 39 A 10

\section{Introduction}

This paper is concerned with the oscillatory properties of solutions of the second order nonlinear difference equation

$$
\Delta^{2} y_{n-1}+\sum_{i=1}^{m} q_{i n} f_{i}\left(y_{n}\right) g_{i}\left(\Delta y_{n-1}\right)=0 \quad(n \in \mathrm{N})
$$

where $\Delta$ is the operator defined by $\Delta y=y_{n+1}-y_{n}, \Delta^{2} y_{n-1}=\Delta\left(\Delta y_{n-1}\right)$ and $\left\{q_{1 n}\right\}, \ldots,\left\{q_{m n}\right\}$ are real sequences. The functions $f_{i}$ and $g_{j}(i=1,2, \ldots, m)$ are defined on the set $\mathbb{R}$ of real numbers. It is interesting to study second order nonlinear difference equations because they are discrete analogues of differential equations. In addition, they do have physical applications as evidenced by $[4,9]$.

By a solution of (1) we mean a real sequence $y=\left\{y_{n}\right\}_{n \geq 0}$ satisfying (1). We consider only such solutions which are nontrivial for all large $n$. A solution of (1) is said to be non-oscillato$7 y$ if it is essentially of constant sign. Otherwise it is called oscillatory. The purpose of this note is to establish some new oscillation criteria (sufficient conditions) for oscillation of all solutions of (1). For some results of this type we refer to the recent papers $[2,3,5-8]$.

Now for the difference equation (1) each result we shall prove requires some of the following conditions, for all $i=1,2, \ldots, m$ :

$\left(C_{1}\right) q_{i n} \geq 0$ for all $n \in N$ and, for every $N \in \mathbb{N}, q_{i n}>0$ for some $n>N$

$\left(C_{2}\right) u f_{j}(u)>0$ and $g_{i}(u)>0$ for $u \neq 0$

(C, $\liminf _{|u| \rightarrow \infty}\left(\left|f_{i}(u)\right| /|u|\right) \geq d>0$

(C. $f_{j}(u)$ is non-decreasing and $f_{i}(-u v) \geq f_{j}(u v) \geq k_{1} f_{j}(u) f_{j}(v)$ for $u, v>0$

(Cs) $g_{i}(u)$ is even and non-increasing for $u>0$

(C. $g_{j}(u)$ is non-increasing and $g_{i}(-u v) \geq g_{i}(u v) \geq k_{2} g_{i}(u) g_{i}(v)$ for $u, v>0$

(C, $f_{i}(u)_{g_{i}}(u) / u^{\alpha} \geq d_{1}>0$ for $u \neq 0$, where $\alpha \in(1, \infty)$. 
In the sequel we need the following two lemmas both due to Hooker and Patula [2].

Lemma 1: If $y_{N} \geq 0, \Delta^{2} y_{n-1} \leq 0$ and $\Delta y_{n}>0$ for $n \geq N$, then $y_{n} \geq n \Delta y_{n-1} / 2$ for all $n \geq 2 N$.

Lemma 2: Assume conditions $\left(\mathrm{C}_{1}\right)$ and $\left(\mathrm{C}_{2}\right)$ and let $y$ be a non-oscillatory solution of (1) such that $y_{n}>0$ for all $n \geq N$, for some $N \geq 0$. Then $y_{n+1}>y_{n}$ and $0<\Delta y_{n+1} \leq \Delta y_{n}$ for all $n \geq$ $N$ (a similar statement holds if $y_{n}$ is essentially negative).

\section{Main rosults}

In this section, we establish sufficient conditions for the oscillation of all solutions of equation (1). We begin with the following

Theorem 1: Let conditions $\left(\mathrm{C}_{1}\right)-\left(\mathrm{C}_{3}\right)$ and $\left(\mathrm{C}_{5}\right)$ hold. If there exists an index $j$ such that

$$
\limsup _{n \rightarrow \infty} n \sum_{l=n}^{\infty} q_{j 1} g_{j}(l)>d^{-1}
$$

where $d$ is as in $\left(\mathrm{C}_{3}\right)$, then equation (1) is oscillatory.

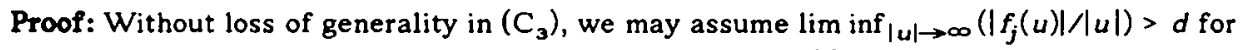
some index $j$. Let $y$ be a non-oscillatory solution of equation (1) and assume without loss of generality that $y_{n}>0$ for all $n \geq N$, for some $N>0$. By Lemma $2, y_{n}$ is increasing and $\Delta y_{n}$ is positive and non-increasing for $n \geq N$. Summing equation (1) from $n$ to $\infty(n \geq N)$, we have

$$
\Delta y_{n-1} \geq \sum_{j=1}^{\infty} q_{j 1} f_{j}\left(y_{1}\right) g\left(\Delta y_{1-1}\right)
$$

Using Lemma 1, we obtain

$$
y_{n} \geq \frac{1}{2} \inf _{1 \geq n} \frac{f_{j}\left(y_{1}\right)}{y_{1}} n \sum_{l=1}^{\infty} q_{j l} g_{j}\left(\Delta y_{1-1}\right) y_{1} \text { for } n \geq 2 N .
$$

Since $y_{n}$ is positive and increasing, $\Delta y_{n}$ is positive and decreasing for $n \geq 2 N$ and using $\left(C_{5}\right)$ the inequality (4) yields

$$
I \geq \frac{1}{2} \inf _{l \geq n} \frac{f_{j}\left(y_{l}\right)}{y_{l}} n \sum_{l=n}^{\infty} q_{j l} g_{j}(l)
$$

This follows since $\Delta y_{n}$ is positive and decreasing for $n \geq 2 N$ and using condition $\left(\mathrm{C}_{s}\right)$ we can take $l$ instead of $\Delta y_{1-1}$ in $g_{j}\left(\Delta y_{1-1}\right)$. From (5), we see that

$$
\frac{1}{d} \geq \frac{1}{2} \inf _{n \rightarrow \infty} n \sum_{=n}^{\infty} q_{j 1} g_{j}(l)
$$

From (6) and (2) we get a contradiction. This completes the proof of the theorem

Remark: Theorem 1 is a discrete analogue of [1:Theorem 1].

Theorem 2: Assume conditions $\left(\mathrm{C}_{1}\right),\left(\mathrm{C}_{2}\right),\left(\mathrm{C}_{6}\right),\left(\mathrm{C}_{7}\right)$ are satisfied. If $\sum_{n=2 N}^{\infty} n q_{j n} g_{j}(2 / n)$ $=\infty$ for some index $j$, then all solutions of equation (1) are oscillatory. 
Proof: Suppose $y$ is a non-oscillatory solution of equation (1) and assume without loss of generality that $y_{n}>0$ for all $n \geq N$, for some $N>0$. By Lemma $2, y_{n}$ is increasing and $\Delta y_{n}$ is positive and non-increasing for $n \geq N$. Using Lemma 1 and $\left(\mathrm{C}_{8}\right)$, we obtain from equation (1)

$$
\Delta^{2} y_{n-1}+k_{2} q_{j n} g_{j}(2 / n) g_{j}\left(y_{n}\right) f_{j}\left(y_{n}\right) \leq 0 \text { for } n \geq 2 N
$$

Multiply both sides of (7) by $n y_{n}^{-\alpha}$ and use $\left(C_{7}\right)$ to obtain

$$
\sum_{n=2 N}^{k-1} n y_{n}^{-\alpha} \Delta^{2} y_{n-1}+\sum_{n=2 N}^{k-1} d_{1} k_{2} n q_{j n} g_{j}(2 / n) \leq 0 \text { for } k \geq 2 N
$$

Upon applying the result

$$
\sum_{i=p}^{n-1} u_{i} \Delta v_{i}=u_{n} v_{n}-u_{p} v_{p}-\sum_{i=p}^{n-1} v_{i+1} \Delta u_{i}
$$

to (8) with $u_{n}=n y_{n}^{-\alpha}$ and $v_{n}=\Delta y_{n-1}$, this yields for any $k \geq 2 N$

$$
k y_{k}^{-\alpha} \Delta y_{k-1}-N y_{2}^{-\alpha} \Delta y_{2 N-1}-\sum_{n=2}^{k-1} \Delta y_{n} \Delta\left(n y_{n}^{-\alpha}\right)+\sum_{n=2 N}^{k-1} d_{1} k_{2} n q_{j n} g_{j}(2 / n) \leq 0 .
$$

In view of Lemma 2 and the hypothesis, (9) implies

$$
\sum_{n=2 N}^{k-1} \Delta y_{n} \Delta\left(n y_{n}^{-\alpha}\right) \rightarrow+\infty \text { as } k \rightarrow \infty .
$$

We shall show that (10) is impossible. For, from (10) we have

$$
\sum_{n=2 N}^{k-1} \Delta y_{n} \Delta\left(n y_{n}^{-\alpha}\right)=\sum_{n=2 N}^{k-1}\left(y_{n+1}^{-\alpha} \Delta y_{n}+n \Delta y_{n} \Delta y_{n}^{-\alpha}\right) \leq \sum_{n=2 N}^{k-1} y_{n+1}^{-\alpha} \Delta y_{n} .
$$

To complete the proof it suffices to show that

$$
\sum_{n=2}^{\infty} y_{n+1}^{-\alpha} \Delta y_{n}<\infty
$$

Let $h(x)=y_{n}+\left(\Delta y_{n}\right)(x-n)(n \leq x \leq n+1, n \geq 2 N)$. Then

$$
h(n)=y_{n}, h(n+1)=y_{n+1} \text { and } h^{\prime}(x)=\Delta y_{n}>0 \quad(n<x<n+1, n \geq 2 N) .
$$

Then $h$ is continuous and increasing for $n \geq 2 N$. We have thus

$$
\begin{aligned}
\sum_{n=2 N}^{k} y_{n+1}^{-\alpha} \Delta y_{n} & =\sum_{n=2 N}^{k} \int_{n}^{n+1} y_{n+1}^{-\alpha} \Delta y_{n} d x \\
& <\sum_{n=2 N}^{k} \int_{n}^{n+1} h^{-\alpha}(x) h^{\prime}(x) d x \leq \frac{1}{1-\alpha}\left(h^{1-\alpha}(k+1)-h^{1-\alpha}(2 N)\right) .
\end{aligned}
$$

Since $\alpha>1$ and $h$ is an increasing function, it follows that (12) holds

Theorem 3: Let conditions $\left(\mathrm{C}_{1}\right),\left(\mathrm{C}_{2}\right)$ and $\left(\mathrm{C}_{4}\right)$ hold and let in addition condition $\left(\mathrm{C}_{7}\right)$ hold for $\alpha \in(0,1)$. If 


$$
\sum_{n=2 N}^{\infty} g_{j n} f_{j}(n / 2)=\infty \text { for some index } j
$$

then all solutions of (1) are oscillatory.

Proof: Suppose $y$ is a non-oscillatory solution of (1) and assume without loss of generality that $y_{n}>0$ for $n \geq N$, for some $N \geq 1$. By Lemma 2, $y_{n}$ is increasing and $\Delta y_{n}$ is positive and non-increasing for $n \geq N$. Dividing (1) by $\left(\Delta y_{n-1}\right)^{\alpha}$ and applying Lemma 1 and using $\left(C_{\downarrow}\right)$ and $\left(C_{1}\right)$ and summing from $2 N$ to $k$, we obtain

$$
\sum_{n=2 N}^{k} \frac{\Delta^{2} y_{n-1}}{\left(\Delta y_{n-1}\right)^{\alpha}}+\sum_{n=2 N}^{k} k_{1} d_{1} q_{j n} f_{j}(n / 2) \leq 0 \text { for } n \geq 2 N
$$

Let $h(x)=y_{n}+\left(\Delta y_{n}\right)(x-n)(n \leq x<n+1, n \geq 2 N)$ as in the proof of Theorem 2. The function $h$ is positive and continuous and increasing since $h^{\prime}(x)=\Delta y_{n}>0(n<x<n+1, n \geq 2 N)$. Let $b(x)$ $=h(x+1)-h(x)>0$. Then $b$ is continuous and non-increasing and $b(x) \leq b(n-1)=\Delta y_{n-1}$. Now for $n-1<x<n$, we have

$$
\frac{\Delta^{2} y_{n-1}}{\left(\Delta y_{n-1}\right)^{\alpha}}=\int_{n-1}^{n} \frac{\Delta^{2} y_{n-1}}{\left(\Delta y_{n-1}\right)^{\alpha}} d x \geq \int_{n-1}^{n} \frac{b^{\prime}(x)}{b^{\alpha}(x)} d x \text {. }
$$

It follows that

$$
\sum_{n=2 N}^{k} \frac{\Delta^{2} y_{n-1}}{\left(\Delta y_{n-1}\right)^{\alpha}} \geq \int_{2 N-1}^{k} \frac{b^{\prime}(x)}{b(x)} d x=\frac{b^{1-\alpha}(k)-b^{1-\alpha}(2 N-1)}{1-\alpha} .
$$

But $b^{1-\alpha}(k)>0$ for all $k>2 N$, so the sum on the left in (15) is bounded below, contradicting (13), which completes the proof

Remark: Theorems 2 and 3 generalize Theorems 4.1 and 4.3, respectively, of Hooker and Patula [2].

Theorem 4: Let conditions $\left(\mathrm{C}_{1}\right),\left(\mathrm{C}_{2}\right)$ and $\left(\mathrm{C}_{6}\right)$ hold and let in addition condition $\left(\mathrm{C}_{7}\right)$ hold for $\alpha=1$. If there exists a positive sequence $\left\{h_{n}\right\}_{n \geq N}$ such that

$$
\sum_{n=2 N}^{\infty} h_{n}\left(\sum_{i=1}^{m} q_{i n} g_{i}(2 / n)-\left(\frac{\Delta h_{n}}{2 h_{n}}\right)^{2}\right)=\infty,
$$

then all solutions of equation (1) are oscillatory.

Proof: Suppose $y$ is a non-oscillatory solution of equation (1) and assume without loss of generality that $y_{n}>0$ for $n \geq N$, for some $N \geq 1$. By Lemma $2, y_{n}$ is increasing and $\Delta y_{n}$ is positive and non-increasing for $n \geq N$. Let us denote $Z_{n}=h_{n} \Delta y_{n-1} / y_{n}(n \geq N)$. Then from equation (1) we get

$$
\Delta Z_{n}=-\frac{h_{n}}{y_{n}} \sum_{i=1}^{m} q_{i n} f_{i}\left(y_{n}\right) g_{i}\left(\Delta y_{n-1}\right)+\frac{\Delta h_{n} \Delta y_{n}}{y_{n+1}}-\frac{h_{n} \Delta y_{n} \Delta y_{n}}{y_{n} y_{n+1}}
$$

In view of monotonicity of $y_{n}$ and $\Delta y_{n},\left(C_{8}\right),\left(C_{7}\right)$ and Lemma 1 we see that

$$
\Delta Z_{n} \leq-d_{1} k_{2} h_{n} \sum_{i=1}^{m} q_{i n} g_{j}(2 / n)+Z_{n+1} \frac{\Delta h_{n}}{h_{n+1}}-Z_{n+1}^{2} \frac{h_{n}}{h_{n+1}^{2}}
$$




$$
=-d_{1} k_{2} h_{n} \sum_{i=1}^{m} q_{i n} g_{i}(2 / n)-\frac{h_{n}}{h_{n+1}^{2}}\left(Z_{n+1}-\frac{\Delta h_{n} h_{n+1}}{2 h_{n}}\right)^{2}+\frac{\left(\Delta h_{n}\right)^{2}}{4 h_{n}} \quad(n \geq 2 N) .
$$

Hence

$$
\Delta Z_{n} \leq-d_{1} k_{2} h_{n} \sum_{i=1}^{m} g_{i n} g_{i}(2 / n)+\frac{\left(\Delta h_{n}\right)^{2}}{4 h_{n}} \quad(n \geq 2 N) .
$$

Summing the above inequality from $2 N$ to $n$, we get

$$
Z_{n+1} \leq Z_{2 N}-d_{1} k_{2} \sum_{i=2 N}^{n} h_{l}\left(\sum_{i=1}^{m} q_{i l} g_{i}(2 / l)-\left(\frac{\Delta h_{l}}{2 h_{1}}\right)^{2}\right)
$$

Now by (16), it is easy to see that $Z_{n}$ is essentially negative, which is a contradiction

\section{REFERENCES}

[1] HAMEDANI, G. G. and G.S. KRENZ: Oscillation criteria for certain socond order difforential equations. J. Math. Anal. Appl. 149 (1990), 271 - 276.

[2] HOOKER, J.W. and W.T. PATULA: A second order nonlinear difference equation: Oscillation and asymptotic behavior. J. Math. Anal. Appl. 91 (1983), 9 - 29.

[3] POPENDA, J.: Oscillation and nonoscillation theoroms for second order differenco equations. J. Math. Anal. Appl. 123 (1987), 34 - 38.

[4] POTTS, R. B.: Exact solution of a difforence approximation to Duffing's oquation. J. Austral. Math. Soc. (Series B) 23 (1981), 64 - 77.

[5] SZMANDA, B.: Oscillation theorems for nonlinear second order difference equations. J. Math. Anal. Appl. 79 (1981), 90 - 95.

[6] SZMANDA, B.: Oscillation criteria for second ordor nonlinear difference oquations. Ann. Polonici Math. 43 (1983), 225 - 235.

[7] SZMANDA, B.: Nonoscillation, oscillation and growth of solutions of nonlinear difference equations of second order. J. Math. Ana1. Appl. 109 (1985), 22 - 30.

[8] THANDAPANI, E.: Oscillatory bohavior of solutions of second ordor nonlinear difforence equations. J. Math. Phys. Sci. (to appear).

[9] WEIL, F.: Existence theorem for the difference equation $y_{n+1}-y_{n}+y_{n-1}=h^{2} f\left(y_{n}\right)$. Intern. J. Math. Sci. 3 (1980), $69-77$.

Received 22.10.1991

E. Thandapani

Madras University P. G. Extn. Centre

Department of Mathematics

Salom-636 O11, Tamil Nadu, India 